\title{
Irinotecan cytotoxicity does not necessarily depend on the UGT1A1 polymorphism but on fluoropyrimidine: A molecular case report
}

\author{
YASUHIRO INOUE $^{1}$, CHIKAO MIKI $^{1}$, HIDEKI WATANABE ${ }^{1}$, JUNICHIRO HIRO ${ }^{1}$, YUJI TOIYAMA ${ }^{1}$, \\ EIKI OJIMA $^{1}$, KANAME NAKATANI ${ }^{2}$, TSUTOMU NOBORI ${ }^{2}$ and MASATO KUSUNOKI ${ }^{1}$
}

\author{
${ }^{1}$ Division of Reparative Medicine, Department of Gastrointestinal and Pediatric Surgery, Institute of Life Sciences, Mie \\ University Graduate School of Medicine; ${ }^{2}$ Department of Laboratory Medicine, Mie University School of Medicine, Japan
}

Received April 25, 2006; Accepted July 12, 2006

\begin{abstract}
We have previously demonstrated that the combined use of doxifluridine and irinotecan shows a different molecular mechanism than that of the protracted venous infusion of 5-FU and irinotecan. In this analysis, there is a suggestion that doxifluridine may enhance irinotecan and enable us to decrease the dose of irinotecan without losing the strong effect by using doxifluridine instead of 5-FU. We present a colon cancer patient with the UGT1A1 polymorphism (UGT1A1*28) as a known high risk for irinotecan, who was treated with a combination of doxifluridine and irinotecan for peritoneal dissemination resulting in stable disease for 2 years without adverse reactions, although the patient initially developed severe adverse effects to the combination of the protracted venous infusion of 5-FU and irinotecan. Even with the same ratios of fluoropyrimidine and irinotecan combinations, replacing 5-FU with doxifluridine or capecitabine could provide new strategies to obtain not only convenience but also better efficacy and safety at the molecular level.
\end{abstract}

\section{Introduction}

We have previously demonstrated that the combined use of doxifluridine and irinotecan shows a different molecular mechanism than that of the protracted venous infusion of 5-FU and irinotecan, even with the same ratio of fluoropyrimidine and irinotecan (1). In the present study, we suggest that doxifluridine may enhance irinotecan action by participating in the effects of the proto-oncogene $c$-fos in tumors. We also suggest that controlling doxifluridine may help in decreasing

Correspondence to: Dr Masato Kusunoki, Division of Reparative Medicine, Department of Gastrointestinal and Pediatric Surgery, Institute of Life Sciences, Mie University Graduate School of Medicine, 2-174 Edobashi, Tsu, Mie 514-8507, Japan

E-mail: kusunoki@clin.medic.mie-u.ac.jp.

Key words: irinotecan, doxifluridine, colon cancer, UGT1A1 the dose of irinotecan, without losing its strong effect, instead of using 5-FU.

Interest in irinotecan for individual treatments has increased since polymorphisms in the metabolizing enzyme UDP-glucuronosyl-transferase 1A1 (UGT1A1) were suggested to be the predictors of response and toxicity in cancer patients (2-4). We present a case of colon cancer with the UGT1A1 polymorphism (UGT1A1*28) treated with a combination of doxifluridine and irinotecan for peritoneal dissemination, resulting in the stability of the disease for 2 years without adverse reactions, although the patient initially developed severe adverse effects to the combination of the protracted venous infusion of 5-FU and irinotecan.

\section{Case report}

In 2003, a 70-year-old woman was diagnosed with ascending colon cancer, and underwent a right side hemicolectomy. A histopathological examination revealed mucinous adenocarcinoma, and no lymph node metastases were found in the resected specimen. The postoperative course was uneventful, and adjuvant chemotherapy was not performed. During a routine post-surgery, serum tumor markers such as the carcinoembryonic antigen (CEA) and CA19-9, which were initially normalized by surgery, increased immediately. Although the patient remained asymptomatic, and the computed tomography was not remarkable, the serum tumor markers had increased progressively. F-18 fluorodeoxyglucose (FDG)-positron emission tomography (FDG-PET) was performed to evaluate occult recurrence. Results showed multiple hot spots in the abdomen and pelvis, and the patient was diagnosed with peritoneal dissemination from ascending colon cancer four months after surgery (Fig. 1).

She was initially treated using Pharmacokinetic modulating chemotherapy (PMC) (5-8). PMC consisted of the continuous intravenous infusion of 5-FU for $24 \mathrm{~h} /$ week, and the oral administration of UFT (Taiho Pharmaceutical Co., Tokyo, Japan) twice a day for 5-7 days/week (5-FU, $600 \mathrm{mg} / \mathrm{m}^{2} / 24 \mathrm{~h}$; UFT, $400 \mathrm{mg} /$ day for a week). Since first-line chemotherapy for recurrent colorectal cancer involves modified PMC using leucovorin ( $\mathrm{LV})$ and irinotecan, the patient was also 


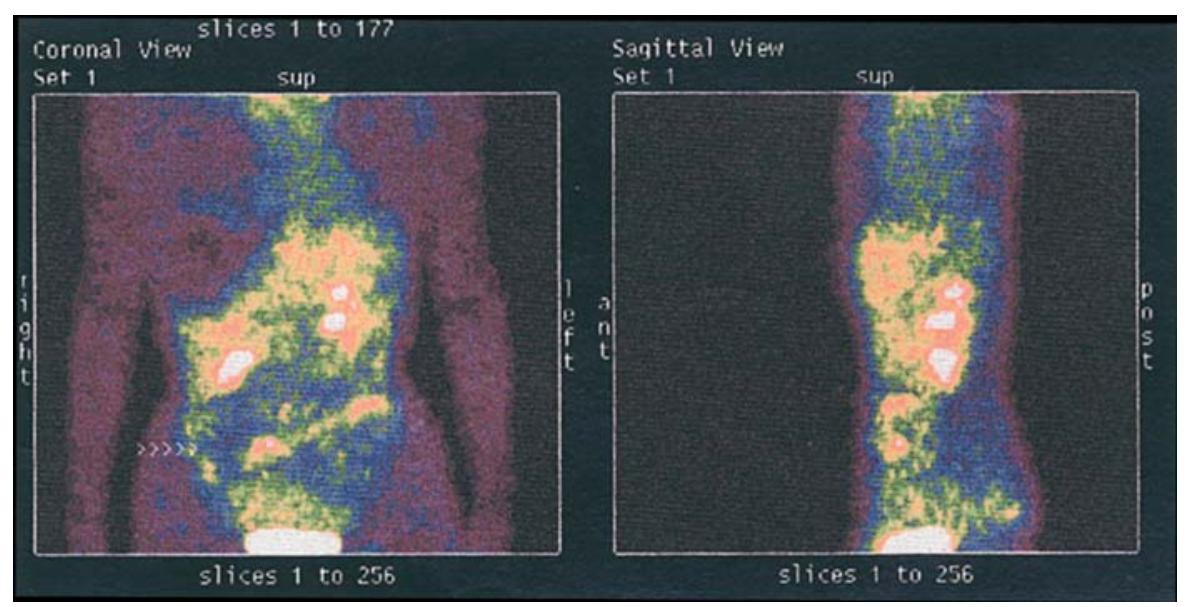

Figure 1. FDG-PET revealing multiple hot spots in the abdomen and pelvis. The patient was diagnosed with peritoneal dissemination from ascending colon cancer.

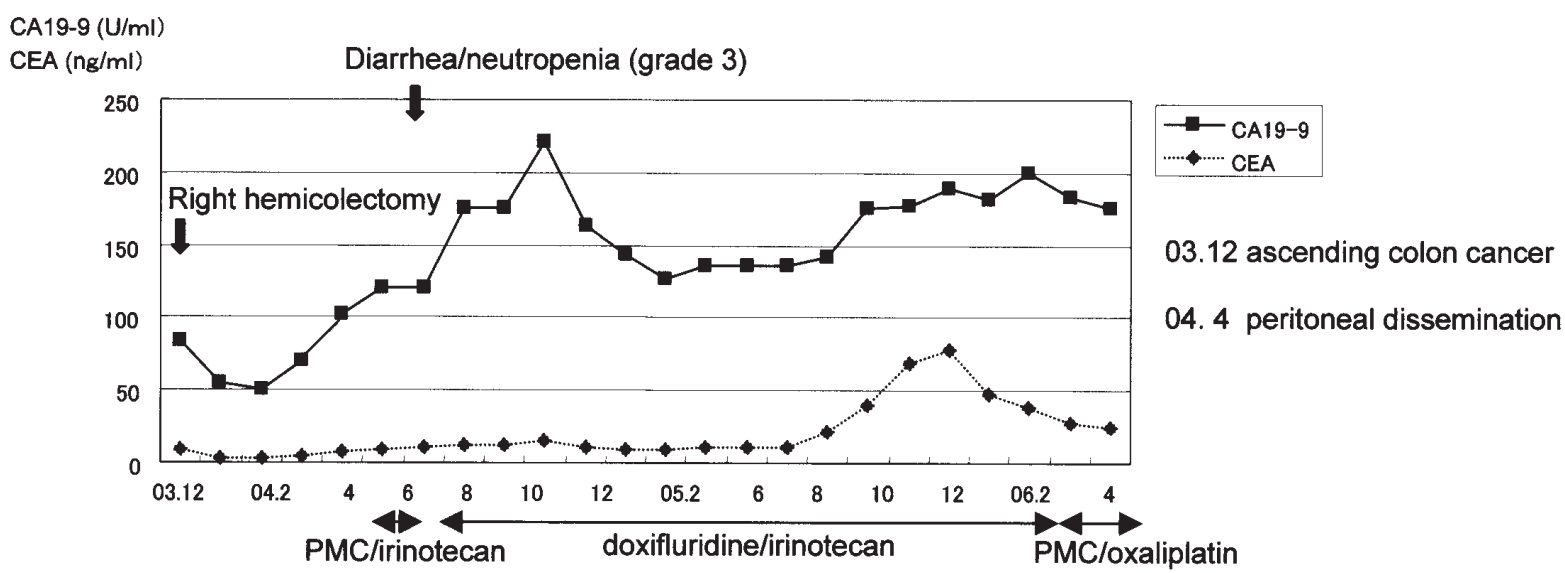

Figure 2. Clinical course and changes in the patient's serum CEA and CA19-9 levels.

administered this modified treatment regimen of PMC (LV, $500 \mathrm{mg} / \mathrm{m}^{2}$; irinotecan, $80 \mathrm{mg} /$ body, biweekly). All toxicities were graded according to the National Cancer Institute Common Toxicity Criteria, Version 2.0 (9). Nine days after the initial administration of irinotecan, the patient experienced severe grade 3 neutropenia and diarrhea. Grade 3 neutropenia $\left(0.5\right.$ to $\left.\leq 1.0 \times 10^{9} / 1\right)$ continued for 3 days after treatment, and then returned to its normal range. Grade 3 diarrhea worsened to 5 stools/day, requiring parental support. We performed systematic management by infusion to regulate water balance for 7 days. Following improvements, we started treatment with a combination of doxifluridine and a low dose of irinotecan. In a previous report, this combination was shown to be promising in patients who were resistant to PMCmodified LV and irinotecan (1). The combination therapy consisted of the continuous intravenous infusion of irinotecan for $24 \mathrm{~h} /$ week, and the oral administration of doxifluridine (irinotecan, $10 \mathrm{mg} /$ body $/ 24 \mathrm{~h}$; doxifluridine, $800 \mathrm{mg} /$ day, for a week). The patient reacted well to this regimen, which was subsequently repeated every week. Consequently, the disease stabilized for about 2 years without adverse reactions (Fig. 2). Although serum CEA and CA19-9 levels remained high, and the patient had a metastatic right ovarian tumor, she underwent chemotherapy at an outpatient clinic, and remains alive for over 2 years after the initial treatment.

\section{Patients and methods}

Patient samples. The patient gave informed written consent for her peripheral blood to be used for research. Variation in UGT1A1 activity most commonly arises from polymorphisms in the UGT1A1 promoter region that contains several repeating TA elements. The presence of 7 TA repeats (referred to as UGT1A1*28), instead of the wild-type number of 6 , results in reduced UGT1A1 expression and activity (10). In addition, UGT1A1*6 and UGT1A1*27, corresponding to variant sequences in exon 1 , have been identified only in Japanese people $(3,10)$. Thus, in this study, we investigated 3 sites of DNA polymorphisms in the UGT1A1 gene (UGT1A $1^{*} 28, \mathrm{UGT} 1 \mathrm{~A} 1{ }^{*} 6$, and UGT1A1*27).

Detection of UGT1A1 gene polymorphisms. Fragments of the promoter and exon 1 containing recurrently screened SNPs of the UGT1A1 gene were amplified by PCR and multiplex PCR. Each PCR mixture (total volume, $10 \mu \mathrm{l}$ ) contained $15 \mathrm{mM}$ Tris- $\mathrm{HCl}$ (pH 8.0), $50 \mathrm{mM} \mathrm{KCl,} 2 \mathrm{mM} \mathrm{MgCl}_{2}, 0.2 \mathrm{mM}$ each of deoxynucleotide triphosphates, $100 \mathrm{ng}$ genomic DNA, $2 \mathrm{U}$ AmpliTaq Gold DNA polymerase (Applied Biosystems), and primers. Following the activation of AmpliTaq Gold DNA polymerase for $10 \mathrm{~min}$ at $95^{\circ} \mathrm{C}$, thermo-cycling conditions were 35 cycles at $94^{\circ} \mathrm{C}$ for $30 \mathrm{sec}, 58^{\circ} \mathrm{C}$ for $30 \mathrm{sec}$, and $72^{\circ} \mathrm{C}$ 
Table I. The UGT1A1*28 polymorphism.

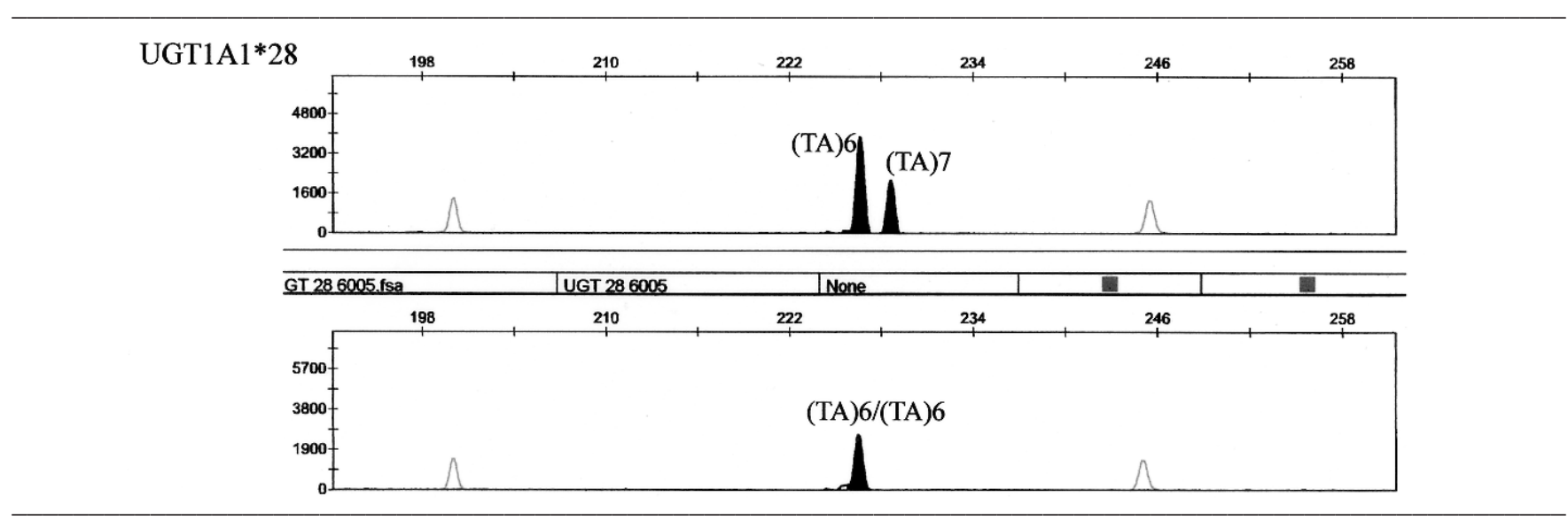

Table II. PCR primers used in this study.

\begin{tabular}{|c|c|c|}
\hline & Forward & Reverse \\
\hline UGT1A $1 * 28$ & FAM-5'-AACTCCCTGCTACCTTTGTGG-3' & 5'-TCTTCCCAGCATGGGACAC-3' \\
\hline UGT1A ${ }^{*} 6$ and 27 & 5'-AAGTAGGAGAGGGCGAACCTC-3' & 5'-GGGCCTAGGGTAATCCTTCAC-3' \\
\hline \multicolumn{3}{|l|}{ SnaPshot Primers } \\
\hline UGT1A1*6 (G211A) & 5'-TTACGCCTCGTTGTACATCAGAGAC-3' & \\
\hline UGT1A1*27(C686A) & 5'-TTTTTTTTCTGTGCGACGTGGTTTATTCCC-3' & \\
\hline
\end{tabular}

for $1 \mathrm{~min}$, with a final elongation step at $72^{\circ} \mathrm{C}$ for $7 \mathrm{~min}$. Fluorescence-labeled PCR products of the promoter region were resolved by capillary electrophoresis on an automated ABI PRISM 310 genetic analyzer (Applied Biosystems). The PCR products of exon 1 containing G211A and C686A were purified using the MiniElute ${ }^{\mathrm{TM}}$ PCR purification kit (Qiagen). Multiplex single nucleotide primer extension analysis reaction was performed with the ABI PRISM SnaPshot multiplex reagent set (Applied Biosystems). The reaction involved the binding of the two primers specific to G211A and C686A to their complementary sequences, in the presence of fluorescence-labeled dideoxynucleotide triphosphates (ddNTPs); and the extension of the primers by AmpliTaq DNA polymerase by adding a single ddNTP to the $3^{\prime}$ end. The reaction mixture contained $0.5 \mu 1 \mathrm{PCR}$ products, $2.5 \mu 1$ multiplex reaction mixture (fluorescence-labeled ddNTPs, AmpliTaq DNA polymerase, and reaction buffer), $0.2 \mathrm{M}$ SnaPshot primers, and $1.8 \mu 1$ distilled water. The reaction was performed as follows: Twenty-five cycles at $96^{\circ} \mathrm{C}$ for $10 \mathrm{sec}, 55^{\circ} \mathrm{C}$ for $5 \mathrm{sec}, 60^{\circ} \mathrm{C}$ for $30 \mathrm{sec}$. Samples were treated with $1 \mathrm{U}$ calf intestinal phosphatase (CIP) at $37^{\circ} \mathrm{C}$ for $1 \mathrm{~h}$, and at $75^{\circ} \mathrm{C}$ for $15 \mathrm{~min}$ to deactivate CIP. Fluorescently labeled fragments were resolved by capillary electrophoresis on an automated ABI PRISM 310 genetic analyzer. The PCR primers are shown in Table I.

\section{Results}

Results showed that the patient did not have UGT1A $1{ }^{*} 6$ and UGT1A $1{ }^{*} 27$, but UGT1A $1{ }^{*} 28$ polymorphisms (Table II).
The patient was heterogeneous for UGT1A1*28, and was more sensitive to the risks of adverse reactions, although previously reported clinical results have been variable for heterogeneous patients compared to homogeneous individuals.

\section{Discussion}

The use of protracted venous infusion of 5-FU combined with irinotecan has become a standard therapy for colorectal cancer (11-13). A current new strategy is to replace 5-FU with an oral agent such as capecitabine, for more convenience with the same efficacy, and for the tolerability of the infusional regimen. A good efficacy of capecitabine combined with irinotecan has been demonstrated in multitude phase II trials (14-16). However, the Japanese national medical insurance has not yet allowed the use of capecitabine for the treatment of colorectal cancer. Since capecitabine is a prodrug of doxifluridine which is approved in Japan, we used doxifluridine and irinotecan combined therapy for colorectal cancer, and recently found two interesting results for the efficacy of this combination (1). i) The combination of doxifluridine and irinotecan showed a different molecular mechanism from that of the protracted venous infusion of 5-FU and irinotecan. ii) This suggested the possibility of decreasing the dose of irinotecan by using doxifluridine instead of 5-FU, to avoid adverse reactions without losing its strong effect. Diarrhea and myelosuppression are the doselimiting toxic effects of irinotecan, and interfere with the optimal utilization of this important drug. Such toxic effects of irinotecan have often been associated with an increased 
level of the active metabolite SN-38. Studies on clinical pharmacogenetics of irinotecan have been mainly focused on the polymorphisms in UGT1A1, the enzyme responsible for the glucuronidation of $\mathrm{SN}-38$ to form the less toxic, inactive metabolic SN-38 glucuronide (15). UGT1A1*28 was shown to be associated with the reduced glucuronidation of $\mathrm{SN}-38$, increased exposure to $\mathrm{SN}-38$, and increased clinical toxicity in patients treated with irinotecan $(3,4,18-20)$. A recent prospective study by Innocenti et al (18) demonstrated that patients with a UGT1A $1 * 28$ allele had a statistically significant high risk of grade 4 neutropenia. A case-control study using Japanese cancer patients also revealed that patients with variant UGT1A1 alleles were at significantly higher risk of severe adverse reactions to irinotecan, suggesting that a genotyping strategy could be clinically useful (3). As a result of these studies, in August 2005, the U.S. Food and Drug Administration (FDA) approved the use of a molecular assay (Invader UGT1A1, made by Third Wave Technologies, Inc.) to identify patients who might be at increased risk of adverse reactions to irinotecan used in the treatment of colorectal cancer. Nevertheless, the clinical importance of pharmacogenetic testing may differ among patient groups, and among clinical situations. In fact, Carlini et al (21) reported that UGT1A1 polymorphisms did not predict response and toxicity in colorectal cancer patients treated with capecitabine and irinotecan in contrast to previous studies. In our case, our patient with UGT1A1*28 was initially treated with a combination of the protracted venous infusion of 5-FU and irinotecan. Consequently, she developed severe adverse effects including grade 3 neutropenia and diarrhea, although she was a heterozygous patient (carrier of one variant allele and one wild-type allele, resulting in intermediate UGT1A1 activity). Accordingly, we introduced a combination of doxifluridine and a low dose of irinotecan as second line chemotherapy, and this resulted in the stability of the disease for 2 years without adverse reactions. Therefore, we confirmed our previous suggestion that doxifluridine might enable us to decrease the dose of irinotecan without losing its strong effect by using a combined therapy.

In conclusion, we reported for the first time that different molecular mechanisms for both efficacy and safety exist, even with the same ratios of fluoropyrimidine and irinotecan combinations. Replacing 5-FU with doxifluridine or capecitabine could provide new strategies to obtain not only convenience but also better efficacy and safety at the molecular level. Although prospective trials are needed to determine the actual clinical benefits, a combination of doxifluridine and irinotecan might prevent patients with UGT1A1 polymorphisms from severe adverse events, and lead to more specific individual patient treatments.

\section{References}

1. Inoue Y, Shirane M, Miki C, et al: Gene expression profiles of colorectal carcinoma in response to neo-adjuvant chemotherapy. Int J Oncol 25: 1641-1649, 2004.

2. Rouits E, Boisdron-Celle M, Dumont A, Guerin O, Morel A and Gamelin E: Relevance of different UGT1A1 polymorphisms in irinotecan-induced toxicity: a molecular and clinical study of 75 patients. Clin Cancer Res 10: 5151-5159, 2004.
3. Ando Y, Saka H, Ando M, et al: Polymorphisms of UDPglucuronosyltransferase gene and irinotecan toxicity: a pharmacogenetic analysis. Cancer Res 60: 6921-6926, 2000.

4. Iyer L, Das S, Janisch L, et al: UGT1A1*28 polymorphism as a determinant of irinotecan disposition and toxicity. Pharmacogenomics J 2: 43-47, 2002.

5. Kusunoki M, Noda M, Yanagi $\mathrm{H}$, Kotera $\mathrm{H}$ and Yamamura $\mathrm{T}$ : Second-look hepatectomy after 5FU arterial infusion in patients with primary unresectable hepatic colorectal metastases: Molecular characteristics of tumors. Int J Oncol 10: 107-111, 1997.

6. Kusunoki M, Yanagi H, Kotera H, Noda M and Yamamura T: Effects of pharmacokinetic modulating chemotherapy using oral UFT and continuous venous 5FU infusion on the prognosis of irradiated rectal carcinomas with p53 overexpression. Int J Oncol 13: 653-657, 1998.

7. Kusunoki M, Yanagi H, Noda M and Yamamura T: The usefulness of pharmacokinetic modulating chemotherapy (UFT plus 5FU) in the treatment of unresectable colorectal carcinomas. Oncol Rep 6: 547-552, 1999.

8. Kusunoki M, Yanagi H, Noda M, Yoshikawa R and Yamamura T: Results of pharmacokinetic modulating chemotherapy in combination with hepatic arterial 5-fluorouracil infusion and oral UFT after resection of hepatic colorectal metastases. Cancer 89: 1228-1235, 2000.

9. National Cancer Institute Common Toxicity Criteria. National Cancer Institute Web site. Available at: http://www.fda.gov/ cder/cancer/toxicityframe.htm. Accessed May 15, 2004.

10. Bosma PJ, Chowdhury JR, Bakker C, et al: The genetic basis of the reduced expression of bilirubin UDP-glucuronosyltransferase 1 in Gilbert's syndrome. N Engl J Med 333: 1171-1175, 1995.

11. Saltz LB, Cox JV, Blanke C, et al: Irinotecan plus fluorouracil and leucovorin for metastatic colorectal cancer. Irinotecan Study Group. N Engl J Med 28: 905-914, 2000.

12. Douillard JY, Cunningham D, Roth AD, et al: Irinotecan combined with fluorouracil compared with fluorouracil alone as first-line treatment for metastatic colorectal cancer: a multicentre randomised trial. Lancet 25: 1041-1047, 2000.

13. Tournigand C, Andre T, Achille E, et al: FOLFIRI followed by FOLFOX 6 or the reverse sequence in advanced colorectal cancer: a randomized GERCOR study. J Clin Oncol 22: 229-237, 2004.

14. Jordan K, Kellner O, Kegel T, Schmoll HJ and Grothey A: Phase II trial of capecitabine/irinotecan and capecitabine/ oxaliplatin in advanced gastrointestinal cancers. Clin Colorectal Cancer 4: 46-50, 2004.

15. Rea DW, Nortier JW, Ten Bokkel Huinink WW, et al: A phase I/II and pharmacokinetic study of irinotecan in combination with capecitabine as first-line therapy for advanced colorectal cancer. Ann Oncol 16: 1123-1132, 2005.

16. Park SH, Bang SM, Cho EK, et al: First-line chemotherapy with irinotecan plus capecitabine for advanced colorectal cancer. Oncology 66: 353-357, 2004.

17. Gupta E, Lestingi TM, Mick R, Ramirez J, Vokes EE and Ratain MJ: Metabolic fate of irinotecan in humans: correlation of glucuronidation with diarrhea. Cancer Res 54: 3723-372, 1994.

18. Innocenti F, Undevia SD, Iyer L, et al: Genetic variants in the UDP-glucuronosyltransferase $1 \mathrm{~A} 1$ gene predict the risk of severe neutropenia of irinotecan. J Clin Oncol 22: 1382-1388, 2004.

19. Iyer L, King CD, Whitington PF, et al: Genetic predisposition to the metabolism of irinotecan (CPT-11). Role of uridine diphosphate glucuronosyltransferase isoform 1A1 in the glucuronidation of its active metabolite $(\mathrm{SN}-38)$ in human liver microsomes. J Clin Invest 101: 847-854,1998.

20. Iyer L, Hall D, Das S, et al: Phenotype-genotype correlation of in vitro $\mathrm{SN}-38$ (active metabolite of irinotecan) and bilirubin glucuronidation in human liver tissue with UGT1A1 promoter polymorphism. Clin Pharmacol Ther 65: 576-582, 1999.

21. Carlini LE, Meropol NJ, Bever J, et al: UGT1A7 and UGT1A9 polymorphisms predict response and toxicity in colorectal cancer patients treated with capecitabine/irinotecan. Clin Cancer Res 11: 1226-1236, 2005. 